\title{
Evaluation of Interdiffusion in Liquid Phase during Reactive Diffusion between $\mathrm{Cu}$ and $\mathrm{Al}$
}

\author{
Yasuhiko Tanaka ${ }^{1}$ and Masanori Kajihara ${ }^{2, *}$ \\ ${ }^{1}$ Graduate School, Tokyo Institute of Technology, Yokohama 226-8502, Japan \\ ${ }^{2}$ Department of Materials Science and Engineering, Tokyo Institute of Technology
}

Using $\mathrm{Cu} / \mathrm{Al}$ diffusion couples initially composed of pure $\mathrm{Cu}$ and $\mathrm{Al}$, the reactive diffusion in the binary $\mathrm{Cu}-\mathrm{Al}$ system was experimentally examined in a previous study. The diffusion couple was isothermally annealed in the temperature range of $T=973-1073 \mathrm{~K}$. Due to annealing, compound layers of the $\beta, \gamma$ and $\varepsilon$ phases are formed between the $\mathrm{Cu}$-rich solid $(\alpha)$ phase and the Al-rich liquid $(L)$ phase, and the $L / \varepsilon$ interface migrates towards the $\varepsilon$ phase. At each annealing time, the migration distance of the $L / \varepsilon$ interface is much greater than the total thickness of the compound layers. Furthermore, there exists the parabolic relationship between the migration distance and the annealing time. This means that the migration of the interface is controlled by the volume diffusion in the $L$ phase. The mathematical model for the interface migration controlled by volume diffusion was used in order to analyze quantitatively the migration rate of the interface. Through the analysis, the interdiffusion coefficient $D$ of the $L$ phase was evaluated to be $1.24 \times 10^{-9}, 2.91 \times 10^{-9}$ and $3.62 \times 10^{-9} \mathrm{~m}^{2} / \mathrm{s}$ at $T=973,1023$ and $1073 \mathrm{~K}$, respectively. Expressing the temperature dependence of $D$ as $D=D_{0} \exp (-Q / R T)$, values of $D_{0}=1.42 \times 10^{-4} \mathrm{~m}^{2} / \mathrm{s}$ and $Q=93.5 \mathrm{~kJ} / \mathrm{mol}$ were obtained by the least-squares method. According to the analysis, the interdiffusion coefficient is much greater for the $L$ phase than for the solid phases. Consequently, the $L / \varepsilon$ interface migrates towards the $\varepsilon$ phase, and the migration rate of the interface is much greater than the overall growth rate of the compound layers. [doi:10.2320/matertrans.47.2480]

(Received June 19, 2006; Accepted August 21, 2006; Published October 15, 2006)

Keywords: intermetallic compounds, bulk diffusion, analytical methods, reactive diffusion, kinetics

\section{Introduction}

There are many binary alloy systems where intermetallic compounds appear as stable phases. ${ }^{1)}$ Reactive diffusion has been experimentally studied for such alloy systems by many investigators. $^{2-31)}$ In those experiments, diffusion couples were prepared from different pure metals or alloys and then isothermally annealed at appropriate temperatures. After annealing, some of the stable compounds were observed as layers at the interface in the diffusion couple. When the reactive diffusion is controlled by volume diffusion, the total thickness $l$ of the compound layers is expressed as a function of the annealing time $t$ by the parabolic relationship $l^{2}=K t$. Here, $K$ is the parabolic coefficient. The parabolic relationship may be believed to hold good in many alloy systems. However, the volume diffusion is not necessarily the ratecontrolling process of reactive diffusion for all the alloy systems.

The reactive diffusion in the binary $\mathrm{Au}-\mathrm{Sn}$ system was experimentally observed using $\mathrm{Sn} / \mathrm{Au} / \mathrm{Sn}$ diffusion couples in previous studies. ${ }^{15-17)}$ The diffusion couple was isothermally annealed at temperatures between $T=393$ and $473 \mathrm{~K}$. Due to annealing, compound layers composed of AuSn, $\mathrm{AuSn}_{2}$ and $\mathrm{AuSn}_{4}$ are produced at the $\mathrm{Au} / \mathrm{Sn}$ interface in the diffusion couple. The total thickness of the compound layers is proportional to a power function of the annealing time, and the exponent of the power function is $0.48,0.42$ and 0.36 at $T=393,433$ and $473 \mathrm{~K}$, respectively. Consequently, the exponent is smaller than 0.5 at most of the annealing temperatures, and thus the parabolic relationship does not hold good for the reactive diffusion in the binary Au-Sn system. This means that grain boundary diffusion as well as volume diffusion contributes to the rate-controlling process

*Corresponding author, E-mail: kajihara@materia.titech.ac.jp and grain growth occurs at certain rates in the compound layers. Such a mixed rate-controlling process was recognized also for the reactive diffusion in the binary $\mathrm{Ag}-\mathrm{Sn},{ }^{18)} \mathrm{Ni}-$ $\mathrm{Sn}^{19)}$ and $\mathrm{Cu}-\mathrm{Sn}^{20)}$ systems.

For the binary $\mathrm{Fe}-\mathrm{Al}$ system, the reactive diffusion was experimentally observed using $\mathrm{Al} / \mathrm{Fe} / \mathrm{Al}$ diffusion couples in a previous study. ${ }^{21)}$ Owing to isothermal annealing at $T=$ $823-913 \mathrm{~K}$, a single compound layer of $\mathrm{Fe}_{2} \mathrm{Al}_{5}$ is formed at the interface in the diffusion couple, and grows according to the parabolic relationship. This indicates that the growth of the $\mathrm{Fe}_{2} \mathrm{Al}_{5}$ layer is controlled by volume diffusion. This type of rate-controlling process was observed also for the binary $\mathrm{Pd}-\mathrm{Sn}$ system. ${ }^{22)}$ In this case, compound layers consisting of $\mathrm{PdSn}_{4}, \mathrm{PdSn}_{3}$ and $\mathrm{PdSn}_{2}$ are formed at $T=433 \mathrm{~K}$, but those composed of only $\mathrm{PdSn}_{4}$ and $\mathrm{PdSn}_{3}$ are produced at $T=453$ and $473 \mathrm{~K}$. At all these temperatures, there exists the parabolic relationship between the total thickness of the $\mathrm{Pd}-\mathrm{Sn}$ compound layers and the annealing time. As previously mentioned, however, the parabolic relationship does not hold good for the binary $\mathrm{Au}-\mathrm{Sn}, \mathrm{Ag}-\mathrm{Sn}, \mathrm{Cu}-\mathrm{Sn}$ and $\mathrm{Ni}-\mathrm{Sn}$ systems. Therefore, the rate-controlling process of reactive diffusion varies depending on the alloy system.

The kinetics of the reactive diffusion controlled by volume diffusion was theoretically analyzed using a mathematical model in a previous study. ${ }^{32)}$ In the theoretical analysis, a hypothetical binary alloy system composed of two primary solid solution phases and one intermetallic compound was considered in order to evaluate the growth rate of the compound in various semi-infinite diffusion couples initially consisting of the two primary solid solution phases with different solubility ranges and interdiffusion coefficients. The mathematical model was also used to analyze numerically the relationship between the temperature dependence of the interdiffusion in each phase and the kinetics of the reactive diffusion. ${ }^{33-35)}$ As mentioned earlier, the single compound 
layer of $\mathrm{Fe}_{2} \mathrm{Al}_{5}$ is formed during reactive diffusion in the binary $\mathrm{Fe}-\mathrm{Al}$ system. ${ }^{21)}$ Furthermore, the growth of the $\mathrm{Fe}_{2} \mathrm{Al}_{5}$ layer is controlled by volume diffusion. Thus, the mathematical model ${ }^{32)}$ was used to analyze numerically the growth behavior of the $\mathrm{Fe}_{2} \mathrm{Al}_{5}$ layer in a previous study. ${ }^{36)}$ Through the analysis, the interdiffusion coefficient of $\mathrm{Fe}_{2} \mathrm{Al}_{5}$ was evaluated quantitatively.

Recently, the reactive diffusion in the binary $\mathrm{Cu}-\mathrm{Al}$ system was experimentally observed using $\mathrm{Cu} / \mathrm{Al}$ diffusion couples by the present authors. ${ }^{28)}$ In that experiment, the diffusion couple was isothermally annealed in the temperature range between $T=973$ and $1073 \mathrm{~K}$. In this temperature range, $\mathrm{Cu}$ is solid, but $\mathrm{Al}$ is liquid. During annealing, compound layers composed of the $\beta, \gamma$ and $\varepsilon$ phases are formed at the interface between the $\mathrm{Cu}$-rich solid $(\alpha)$ phase and the Al-rich liquid $(L)$ phase. The total thickness $l$ of the compound layers is proportional to a power function of the annealing time $t$, and the exponent of the power function is $0.15,0.41$ and 0.33 at $T=973,1023$ and $1073 \mathrm{~K}$, respectively. Hence, the exponent is smaller than 0.5 at all the annealing temperatures. As a consequence, like the binary $\mathrm{Au}-\mathrm{Sn},{ }^{15-17)} \mathrm{Ag}-\mathrm{Sn},{ }^{18)} \mathrm{Ni}-\mathrm{Sn}^{19)}$ and $\mathrm{Cu}-\mathrm{Sn}^{20)}$ systems, grain boundary diffusion as well as volume diffusion contributes to the rate-controlling process and grain growth takes place at certain rates in the compound layers for the binary $\mathrm{Cu}-\mathrm{Al}$ system. Unfortunately, however, the mathematical model ${ }^{32)}$ mentioned above cannot be applicable to such a ratecontrolling process. On the other hand, the interface between the $L$ and $\varepsilon$ phases migrates towards the $\varepsilon$ phase during reactive diffusion in the binary $\mathrm{Cu}-\mathrm{Al}$ system. Furthermore, the migration distance $w$ of the $L / \varepsilon$ interface is much greater than the total thickness $l$ of the compound layers, and the square of the migration distance $w$ is proportional to the annealing time $t$. This means that interdiffusion occurs more remarkably in the $L$ phase than in the $\alpha, \beta, \gamma$ and $\varepsilon$ phases, and the migration of the $L / \varepsilon$ interface is controlled by the volume diffusion in the $L$ phase. In the present study, the interdiffusion coefficient $D$ for volume diffusion in the $L$ phase of the binary $\mathrm{Cu}-\mathrm{Al}$ system was quantitatively evaluated from the experimental result for the migration behavior of the $L / \varepsilon$ interface.

\section{Experimental Summary}

As mentioned in Section 1, the reactive diffusion in the binary $\mathrm{Cu}-\mathrm{Al}$ system was experimentally observed in a previous study. ${ }^{28)}$ In that experiment, columnar diffusion couples consisting of pure solid $\mathrm{Cu}$ and pure liquid $\mathrm{Al}$ were isothermally annealed in the temperature range between $T=$ 973 and $1073 \mathrm{~K}$. Here, the diameter is 8 and $8.5 \mathrm{~mm}$ for $\mathrm{Cu}$ and $\mathrm{Al}$, respectively, and the initial thickness is 5 and $4.8 \mathrm{~mm}$ for $\mathrm{Cu}$ and $\mathrm{Al}$, respectively. The interface between $\mathrm{Cu}$ and $\mathrm{Al}$ is flat and perpendicular to the columnar axis. During annealing, compound layers of the $\beta, \gamma$ and $\varepsilon$ phases are produced at the interface in the $\mathrm{Cu} / \mathrm{Al}$ diffusion couple. According to a recent phase diagram in the binary $\mathrm{Cu}-\mathrm{Al}$ system, ${ }^{37)}$ the $\beta, \gamma$ and $\varepsilon$ phases are the only stable compounds at $T=973-1073 \mathrm{~K}$. At these temperatures, the $\beta$ and $\varepsilon$ phases are in equilibrium with the $\mathrm{Cu}$-rich solid $(\alpha)$ and Al-rich liquid $(L)$ phases, respectively. Consequently, all

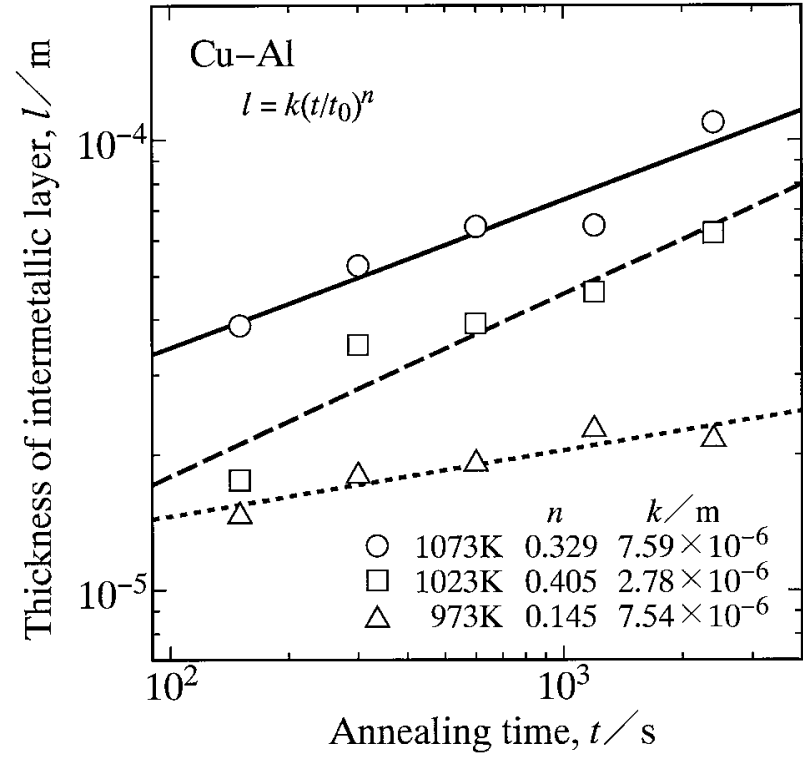

Fig. 1 The total thickness $l$ of the intermetallic layer versus the annealing time $t$ shown as open triangles, squares and circles at $T=973,1023$ and $1073 \mathrm{~K}$, respectively. Straight lines indicate the calculations from eq. (1).

the stable phases were recognized in the annealed $\mathrm{Cu} / \mathrm{Al}$ diffusion couple. The layer composed of the $\beta, \gamma$ and $\varepsilon$ phases is hereafter called the intermetallic layer. The total thickness $l$ of the intermetallic layer was determined experimentally. The values of $l$ are plotted against the annealing time $t$ in Fig. 1. In this figure, the ordinate and the abscissa show the logarithms of $l$ and $t$, respectively. Furthermore, open triangles, squares and circles indicate the results of $T=973$, 1023 and $1073 \mathrm{~K}$, respectively. As can be seen, the thickness $l$ monotonically increases with increasing annealing time $t$. The plotted points at each annealing temperature are located well on a straight line. This yields that the thickness $l$ is expressed as a power function of the annealing time $t$ by the equation

$$
l=k\left(t / t_{0}\right)^{n} .
$$

Here, $t_{0}$ is unit time, $1 \mathrm{~s}$. It is adopted to make the argument $t / t_{0}$ of the power function dimensionless. The proportionality coefficient $k$ has the same dimension as the thickness $l$, but the exponent $n$ is dimensionless. From the plotted points in Fig. 1, the values of $k$ and $n$ were determined by the leastsquares method. The determined values are shown in Fig. 1. Using these values of $k$ and $n$, the thickness $l$ was calculated as a function of the annealing time $t$ from eq. (1). The results of $T=973,1023$ and $1073 \mathrm{~K}$ are indicated as dotted, dashed and solid lines, respectively, in Fig. 1. At each experimental annealing time, the thickness $l$ monotonically increases with increasing annealing temperature $T$. Thus, the higher the annealing temperature $T$ is, the faster the intermetallic layer grows.

According to the result in Fig. 1, the exponent $n$ is 0.15 , 0.41 and 0.33 at $T=973,1023$ and $1073 \mathrm{~K}$, respectively. If the volume diffusion of the constituent elements in each phase is the rate-controlling process for the growth of the intermetallic layer, $n$ is equal to 0.5 . On the other hand, the growth will be controlled by the grain boundary diffusion 
along grain boundaries with a finite thickness in the intermetallic layer at annealing temperatures where the volume diffusion is much slower than the grain boundary diffusion. When grain growth occurs in the intermetallic layer, the volume fraction of the grain boundaries monotonically decreases with increasing annealing time. Such decrease in the volume fraction causes the decrement of the effective cross-section, and decelerates the grain boundary diffusion. As a result, $n$ becomes smaller than $0.5{ }^{38)}$ When the grain growth occurs very sluggishly, the volume fraction of the grain boundaries remains almost constant during annealing. In such a case, the effective cross-section for the grain boundary diffusion hardly varies, and thus $n$ is almost equal to 0.5. According to the result in Fig. 1, $n$ is smaller than 0.5 at all the annealing temperatures. Consequently, it is concluded that the grain boundary diffusion as well as the volume diffusion contributes to the rate-controlling process and the grain growth occurs at certain rates in the intermetallic layer for the reactive diffusion in the binary $\mathrm{Cu}-\mathrm{Al}$ system at $T=973-1073 \mathrm{~K}$.

During growth of the intermetallic layer, the interface between the $L$ and $\varepsilon$ phases migrates towards the $\varepsilon$ phase. Subtracting the thicknesses of the intermetallic layer and the $\alpha$ phase from the initial thickness of the $\alpha$ phase, we can determine the migration distance $w$ of the $L / \varepsilon$ interface at each annealing time. ${ }^{28)}$ The values of $w$ are plotted against the annealing time $t$ in Fig. 2. In this figure, the ordinate indicates the migration distance $w$, and the abscissa shows the square root of the annealing time $t$. Open triangles, squares and circles indicate the results of $T=973,1023$ and $1073 \mathrm{~K}$, respectively. Although the open triangles are rather scattered, most of the plotted points lie well on the corresponding straight line. This means that the parabolic relationship holds good between $w$ and $t$ as follows.

$$
w^{2}=K t
$$

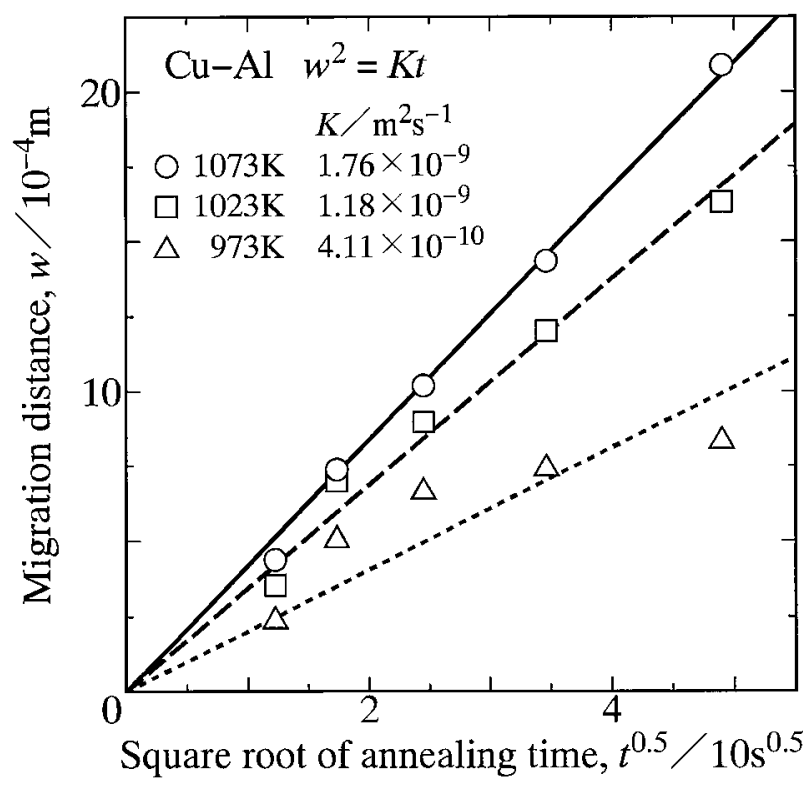

Fig. 2 The migration distance $w$ of the $L / \varepsilon$ interface versus the square root of the annealing time $t$ shown as open triangles, squares and circles at $T=973,1023$ and $1073 \mathrm{~K}$, respectively. Straight lines indicate the calculations from eq. (2).
Here, $K$ is the parabolic coefficient with a dimension of $\mathrm{m}^{2} / \mathrm{s}$. From the plotted points in Fig. 2, the value of $K$ was evaluated at each annealing temperature by the least-squares method. The evaluated values of $K$ are shown in Fig. 2. Using these values of $K, w$ was calculated as a function of $t$ from eq. (2). The results of $T=973,1023$ and $1073 \mathrm{~K}$ are indicated as dotted, dashed and solid lines, respectively, in Fig. 2.

The ratio $r$ of the thickness $l$ to the migration distance $w$ is defined as

$$
r=l / w
$$

The values of $r$ are plotted against the annealing time $t$ in Fig. 3. In this figure, the ordinate shows the ratio $r$, and the abscissa indicates the logarithm of the annealing time $t$. Open triangles, squares and circles show the results of $T=973$, 1023 and $1073 \mathrm{~K}$, respectively. As can be seen, the ratio $r$ takes values between 0.03 and 0.08 under the present annealing conditions. Although the plotted points are slightly scattered in Fig. 3, various straight lines indicate that $r$ is a monotonically decreasing function of $t$. Consequently, we may conclude that the thickness $l$ is much smaller than the migration distance $w$ even at longer annealing times.

The values of $K$ are plotted against the annealing temperature $T$ as open circles in Fig. 4. In this figure, the ordinate shows the logarithm of $K$, and the abscissa indicates the reciprocal of $T$. If the temperature dependence of $K$ is expressed by the equation

$$
K=K_{0} \exp \left(-Q_{K} / R T\right)
$$

the pre-exponential factor $K_{0}$ and the activation enthalpy $Q_{K}$ are evaluated to be $3.01 \times 10^{-3} \mathrm{~m}^{2} / \mathrm{s}$ and $127 \mathrm{~kJ} / \mathrm{mol}$, respectively, from the open circles in Fig. 4 by the leastsquares method. Here, $R$ is the gas constant. Using these parameters, $K$ was calculated as a function of $T$ from eq. (4). The result is shown as a solid line in Fig. 4.

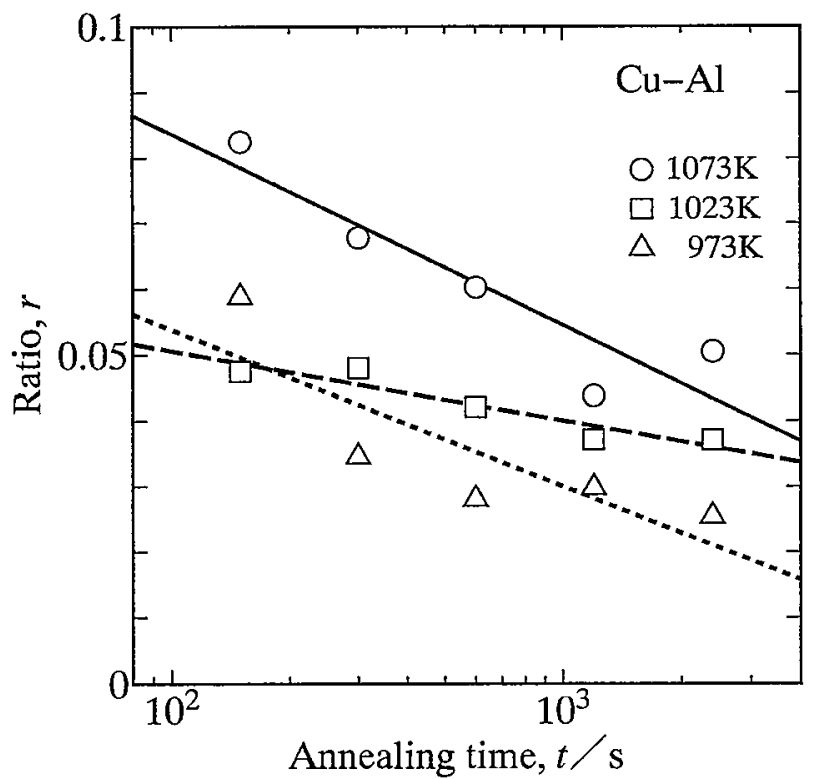

Fig. 3 The ratio $r$ versus the annealing time $t$ shown as open triangles, squares and circles at $T=973,1023$ and $1073 \mathrm{~K}$, respectively. 


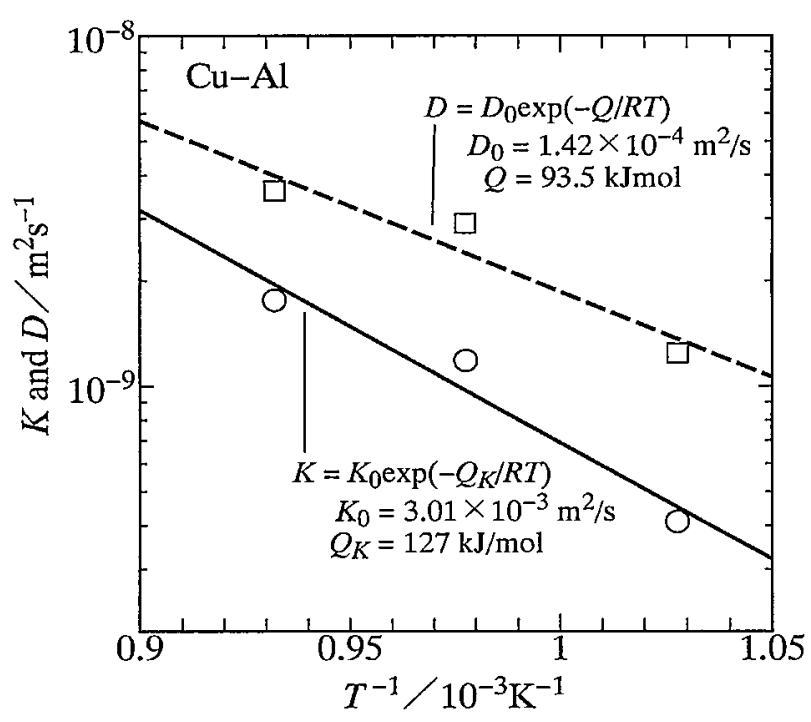

Fig. 4 The parabolic coefficient $K$ versus the reciprocal of the annealing temperature $T$ shown as open circles. The evaluations for the interdiffusion coefficient $D$ of the $L$ phase are indicated as open squares. Solid and dashed lines show the calculations from eqs. (4) and (17), respectively.

\section{Model}

As mentioned in Section 2, the thickness $l$ of the intermetallic layer is much smaller than the migration distance $w$ of the $L / \varepsilon$ interface. This means that interdiffusion occurs less remarkably in the $\alpha, \beta, \gamma$ and $\varepsilon$ phases than in the $L$ phase. In such a case, the migration rate of the $L / \varepsilon$ interface is predominantly determined by the interdiffusion in the $L$ phase, and thus can be described by a simple mathematical model. This model will be explained briefly below.

Let us consider a semi-infinite diffusion couple composed of the $\alpha$ and $\beta$ phases with initial compositions of $c^{\alpha 0}$ and $c^{\beta 0}$, respectively. Here, the $\alpha$ and $\beta$ phases are the A-rich and Brich phases, respectively, in a binary A-B system, and $c$ is the concentration of element B measured in mol per unit volume. In the semi-infinite diffusion couple, the thickness is semiinfinite for the $\alpha$ and $\beta$ phases, and the $\alpha / \beta$ interface is flat. Therefore, the interdiffusion of elements A and B occurs unidirectionally along the direction perpendicular to the flat interface. This direction is called the diffusional direction. If the diffusion couple is annealed at temperature $T$ for an appropriate time, the $\alpha / \beta$ interface will migrate towards the $\alpha$ or $\beta$ phase depending on the flux balance at the interface. The concentration profile of element $\mathrm{B}$ along the diffusional direction across the $\alpha / \beta$ interface is schematically drawn in Fig. 5. In this figure, the ordinate shows the concentration $c$, and the abscissa indicates the distance $x$ measured from the initial position of the $\alpha / \beta$ interface. When the migration of the $\alpha / \beta$ interface is controlled by the volume diffusion in the $\alpha$ and $\beta$ phases and the molar volumes of these phases are equivalent each other, the migration rate $\mathrm{d} w / \mathrm{d} t$ of the interface is related to the flux balance at the interface by the equation $^{39)}$

$$
\left(c^{\beta \alpha}-c^{\alpha \beta}\right) \frac{\mathrm{d} w}{\mathrm{~d} t}=J^{\beta \alpha}-J^{\alpha \beta}
$$

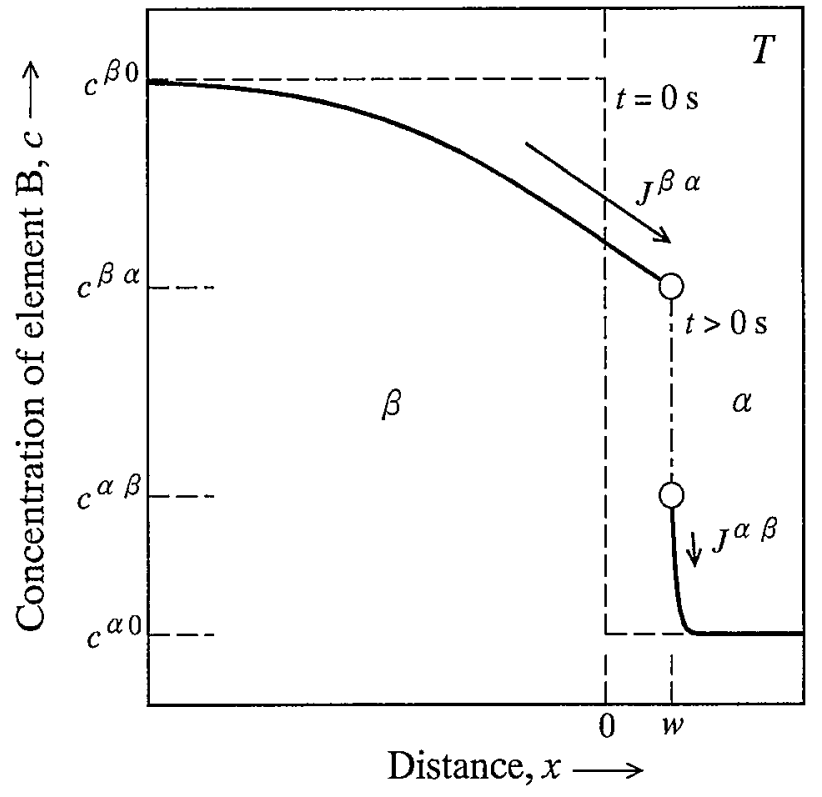

Fig. 5 Concentration profile of element B across the $\alpha / \beta$ interface along the diffusional direction in the semi-infinite diffusion couple.

Here, $w$ is the migration distance of the $\alpha / \beta$ interface measured from the origin of the distance $x, c^{\alpha \beta}$ and $c^{\beta \alpha}$ are the compositions of the $\alpha$ and $\beta$ phases, respectively, at the interface, and $J^{\alpha \beta}$ and $J^{\beta \alpha}$ are the diffusional fluxes of element $\mathrm{B}$ due to the volume diffusion in the $\alpha$ and $\beta$ phases, respectively, at the interface. According to Fick's first law, the diffusional flux $J^{\theta}$ is proportional to the concentration gradient $\partial c^{\theta} / \partial x$ as follows.

$$
J^{\theta}=-D^{\theta} \frac{\partial c^{\theta}}{\partial x} \quad(\theta=\alpha, \beta)
$$

In eq. (6), $D^{\theta}$ is the interdiffusion coefficient for the volume diffusion in the $\theta$ phase, where $\theta$ stands for $\alpha$ and $\beta$. When the interdiffusion coefficient $D^{\theta}$ is independent of the composition of the $\theta$ phase, Fick's second law is expressed as follows.

$$
\frac{\partial c^{\theta}}{\partial t}=D^{\theta} \frac{\partial^{2} c^{\theta}}{\partial x^{2}} \quad(\theta=\alpha, \beta)
$$

Equation (7) shows that the composition $c^{\theta}$ is a function of the distance $x$ and the annealing time $t$. For the semi-infinite $\alpha / \beta$ diffusion couple, the initial conditions are described as

$$
c^{\alpha}(x>0, t=0)=c^{\alpha 0}
$$

and

$$
c^{\beta}(x<0, t=0)=c^{\beta 0},
$$

and the boundary conditions are expressed by the equations

$$
\begin{aligned}
& c^{\alpha}(x=w, t>0)=c^{\alpha \beta}, \\
& c^{\beta}(x=w, t>0)=c^{\beta \alpha}, \\
& c^{\alpha}(x=+\infty, t>0)=c^{\alpha 0}
\end{aligned}
$$

and

$$
c^{\beta}(x=-\infty, t>0)=c^{\beta 0} .
$$


Under these initial and boundary conditions, eqs. (5)-(7) are solved analytically. If $D^{\alpha}$ is much smaller than $D^{\beta}, J^{\alpha \beta}$ becomes negligible compared with $J^{\beta \alpha}$ according to eq. (6) unless $\partial c^{\beta} / \partial x$ is much smaller than $\partial c^{\alpha} / \partial x$. In such a case, the following equation is obtained from eqs. (5) and (6).

$$
\left(c^{\beta \alpha}-c^{\alpha \beta}\right) \frac{\mathrm{d} w}{\mathrm{~d} t}=J^{\beta \alpha}=-\left.D^{\beta} \frac{\partial c^{\beta}}{\partial x}\right|_{x=w}
$$

For the migration of the $\alpha / \beta$ interface controlled by the volume diffusion, the migration distance $w$ is expressed as a function of the annealing time $t$ by the equation ${ }^{39)}$

$$
w=K_{w} \sqrt{4 D^{\beta} t} \text {. }
$$

Here, $K_{w}$ is the dimensionless coefficient. The coefficient $K_{w}$ is related with the initial and boundary conditions in eqs. (8) and (9) as follows. ${ }^{39)}$

$$
\frac{\exp \left\{-\left(K_{w}\right)^{2}\right\}}{K_{w}\left\{1+\operatorname{erf}\left(K_{w}\right)\right\}}=\frac{c^{\beta \alpha}-c^{\alpha 0}}{c^{\beta 0}-c^{\beta \alpha}} \sqrt{\pi}
$$

On the other hand, the following equation is obtained from eqs. (2) and (11).

$$
w^{2}=4 D^{\beta}\left(K_{w}\right)^{2} t=K t
$$

Equation (13) shows that the parabolic coefficient $K$ is expressed as a function of the interdiffusion coefficient $D^{\beta}$ of the $\beta$ phase and the dimensionless coefficient $K_{w}$ by the equation

$$
K=4 D^{\beta}\left(K_{w}\right)^{2} .
$$

Since $K_{w}$ is dimensionless, the dimension of $K$ corresponds with that of $D^{\beta}$ according to eq. (14). Furthermore, $K_{w}$ is a function of $c^{\alpha 0}, c^{\beta \alpha}$ and $c^{\beta 0}$ through eq. (12), and hence $K$ becomes a function of $c^{\alpha 0}, c^{\beta \alpha}, c^{\beta 0}$ and $D^{\beta}$ via eq. (14). Thus, $D^{\beta}$ is an inverse function of $c^{\alpha 0}, c^{\beta \alpha}, c^{\beta 0}$ and $K$. Consequently, $D^{\beta}$ can be evaluated from the value of $K$ determined experimentally for given values of $c^{\alpha 0}, c^{\beta \alpha}$ and $c^{\beta 0}$.

\section{Results and Discussion}

\subsection{Evaluation of interdiffusion}

In the mathematical model mentioned in Section 3, the composition is described with the concentration $c$ of element $\mathrm{B}$ measured in mol per unit volume. On the other hand, the mol fraction $y$ of element $\mathrm{B}$ is practically used to express the composition of each phase. However, the mol fraction $y$ is readily converted into the concentration $c$ by the equation $c=y / V_{\mathrm{m}}$. Here, $V_{\mathrm{m}}$ is the molar volume of the relevant phase. When the molar volume $V_{\mathrm{m}}$ is constant independently of the composition at each annealing temperature, the concentrations $c^{\alpha 0}, c^{\beta \alpha}$ and $c^{\beta 0}$ in eq. (12) are automatically replaced with the mol fractions $y^{\alpha 0}, y^{\beta \alpha}$ and $y^{\beta 0}$, respectively. Here, the superscript of the mol fraction $y$ possesses the same meaning as the concentration $c$. In the schematic concentration profile of Fig. 5, the diffusional flux $J^{\theta}$ is much greater in the $\beta$ phase than in the $\alpha$ phase, and thus the $\alpha / \beta$ interface migrates towards the $\alpha$ phase. Consequently, the $\mathrm{Cu}$-rich $\alpha$ and Al-rich $L$ phases in the $\mathrm{Cu} / \mathrm{Al}$ diffusion couple correspond to the $\alpha$ and $\beta$ phases, respectively, for the mathematical model in Section 3. However, the $L$ phase is actually in equilibrium with the $\varepsilon$ phase at each annealing

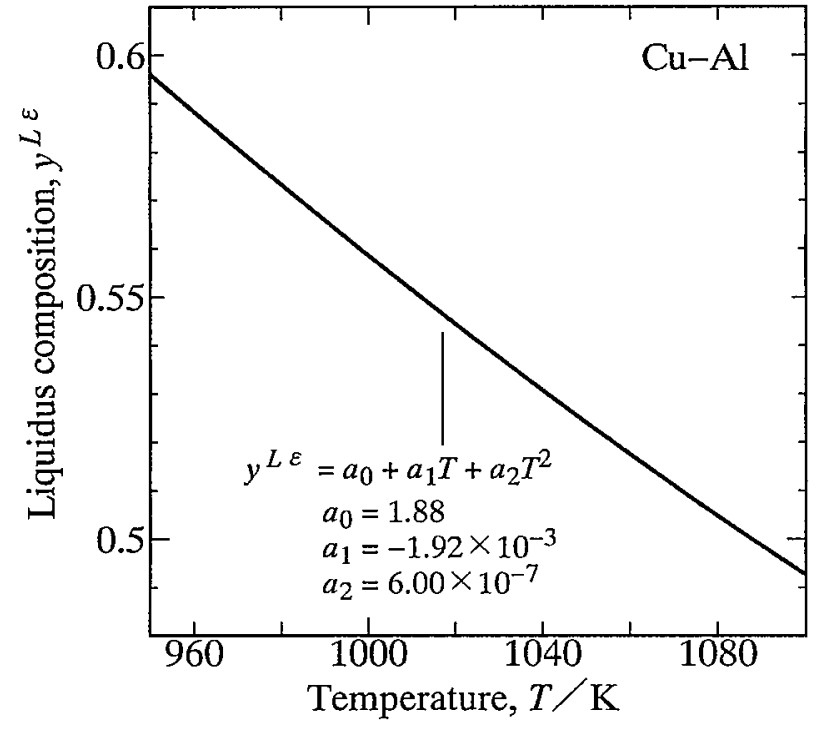

Fig. 6 The liquidus composition $y^{L \varepsilon}$ versus the temperature $T .{ }^{37)}$

temperature, and thus the liquidus composition corresponds to the mol fraction $y^{L \varepsilon}$ of the $L$ phase for the $L+\varepsilon$ two-phase tie-line. As a result, we obtain the following equation.

$$
\begin{aligned}
& \left(y^{L \varepsilon}-y^{\alpha 0}\right) \sqrt{\pi}\left\{1+\operatorname{erf}\left(K_{w}\right)\right\} K_{w} \\
& \quad-\left(y^{L 0}-y^{L \varepsilon}\right) \exp \left\{-\left(K_{w}\right)^{2}\right\}=0
\end{aligned}
$$

Here, $y^{\alpha 0}$ and $y^{L 0}$ are the initial compositions of the $\alpha$ and $L$ phases, respectively. From eq. (15), $K_{w}$ is calculated for given values of $y^{\alpha 0}, y^{L \varepsilon}$ and $y^{L 0}$. Since $K_{w}$ is an implicit function of $y^{\alpha 0}, y^{L \varepsilon}$ and $y^{L 0}$, however, the calculation cannot be carried out in an explicit manner. Therefore, NewtonRaphson's method ${ }^{40)}$ was used to calculate numerically the value of $K_{w}$ from eq. (15). According to a recent phase diagram in the binary $\mathrm{Cu}-\mathrm{Al}$ system, ${ }^{37)} y^{L \varepsilon}=0.579,0.542$ and 0.509 at $T=973,1023$ and $1073 \mathrm{~K}$, respectively. If the temperature dependence of the composition $y^{L \varepsilon}$ is described by the equation ${ }^{36)}$

$$
y^{L \varepsilon}=a_{0}+a_{1} T+a_{2} T^{2},
$$

the following values are obtained: $a_{0}=1.88, a_{1}=-1.92 \times$ $10^{-3}$ and $a_{2}=6.00 \times 10^{-7}$. Using these parameters, the temperature dependence of $y^{L \varepsilon}$ was calculated from eq. (16). The result is shown as a solid curve in Fig. 6. Furthermore, $y^{\alpha 0}=0$ and $y^{L 0}=1$ for the $\mathrm{Cu} / \mathrm{Al}$ diffusion couple. For these values of $y^{\alpha 0}, y^{L \varepsilon}$ and $y^{L 0}$, the calculation provides $K_{w}=0.288,0.319$ and 0.349 at $T=973,1023$ and $1073 \mathrm{~K}$, respectively. On the other hand, as shown in Fig. 2, $K=4.11 \times 10^{-10}, 1.18 \times 10^{-9}$ and $1.76 \times 10^{-9} \mathrm{~m}^{2} / \mathrm{s}$ were experimentally determined at $T=973,1023$ and $1073 \mathrm{~K}$, respectively. Inserting these values of $K$ and $K_{w}$ into eq. (14), we finally obtain $D=1.24 \times 10^{-9}, 2.91 \times 10^{-9}$ and $3.62 \times$ $10^{-9} \mathrm{~m}^{2} / \mathrm{s}$ at $T=973,1023$ and $1073 \mathrm{~K}$, respectively, for the $L$ phase in the binary $\mathrm{Cu}-\mathrm{Al}$ system. The values of $D$ are plotted against the reciprocal of $T$ as open squares in Fig. 4. The temperature dependence of $D$ is usually described by the following equation of the same formula as eq. (4).

$$
D=D_{0} \exp (-Q / R T)
$$

Here, $D_{0}$ is the pre-exponential factor, and $Q$ is the activation 
enthalpy. From the open squares in Fig. $4, D_{0}=1.42 \times$ $10^{-4} \mathrm{~m}^{2} / \mathrm{s}$ and $Q=93.5 \mathrm{~kJ} / \mathrm{mol}$ are evaluated by the leastsquares method. Using these parameters, $D$ was calculated as a function of $T$ from eq. (17). The result is shown as a dashed line in Fig. 4. As can be seen, the absolute value is slightly greater for $D$ than for $K$ at each annealing temperature. Sometimes, $D$ may be roughly estimated from $K$ by the equation

$$
D=f K,
$$

where $f$ takes a constant value of $1,0.5$ or 0.25 . This estimation insists that $D$ is not greater than $K$. However, $D$ is greater than $K$ in Fig. 4, and thus $f$ is greater than unity in eq. (18). Furthermore, $Q$ is smaller than $Q_{K}$, and hence $f$ varies depending on the temperature. Therefore, there is no adequate way to estimate the temperature dependence of $f$. Consequently, the interdiffusion coefficient $D$ cannot be necessarily estimated only from the parabolic coefficient $K$ in a straightforward manner.

The temperature dependence of the tracer diffusion coefficient $D_{i}^{*}(i=\mathrm{Cu}, \mathrm{Al})$ is also expressed by the equation of the same formula as eq. (17) with the pre-exponential factor $D_{i 0}^{*}$ and the activation enthalpy $Q_{i}^{*}$. Unfortunately, however, reliable information is not available for $D_{\mathrm{A} 10}^{*}$ and $Q_{\mathrm{Al}}^{*}$ of the tracer diffusion coefficient $D_{\mathrm{Al}}^{*}$ of $\mathrm{Al}$ in the $L$ phase of pure $\mathrm{Cu}$. On the other hand, Ejima and co-workers ${ }^{41}$ ) determined values of $D_{\mathrm{Cu} 0}^{*}=1.05 \times 10^{-7} \mathrm{~m}^{2} / \mathrm{s}$ and $Q_{\mathrm{Cu}}^{*}=$ $23.8 \mathrm{~kJ} / \mathrm{mol}$ for the tracer diffusion coefficient $D_{\mathrm{Cu}}^{*}$ of $\mathrm{Cu}$ in the $L$ phase of pure Al. Using these values of $D_{\mathrm{Cu} 0}^{*}$ and $Q_{\mathrm{Cu}}^{*}$, $D_{\mathrm{Cu}}^{*}$ was calculated as a function of the temperature $T$ from eq. (17). The result is shown as a dashed line in Fig. 7. In this figure, the ordinate indicates the logarithm of $D_{\mathrm{Cu}}^{*}$, and the abscissa shows the reciprocal of $T$. The dashed line for $D$ in Fig. 4 is indicated again as a solid line in Fig. 7. As can be seen, $D$ is close to $D_{\mathrm{Cu}}^{*}$ at $T=973-1073 \mathrm{~K}$.

The solid-state reactive diffusion in the binary $\mathrm{Cu}-\mathrm{Al}$ system was experimentally observed using $\mathrm{Al} / \mathrm{Cu} / \mathrm{Al}$ diffusion couples by Funamizu and Watanabe. ${ }^{42)}$ In their

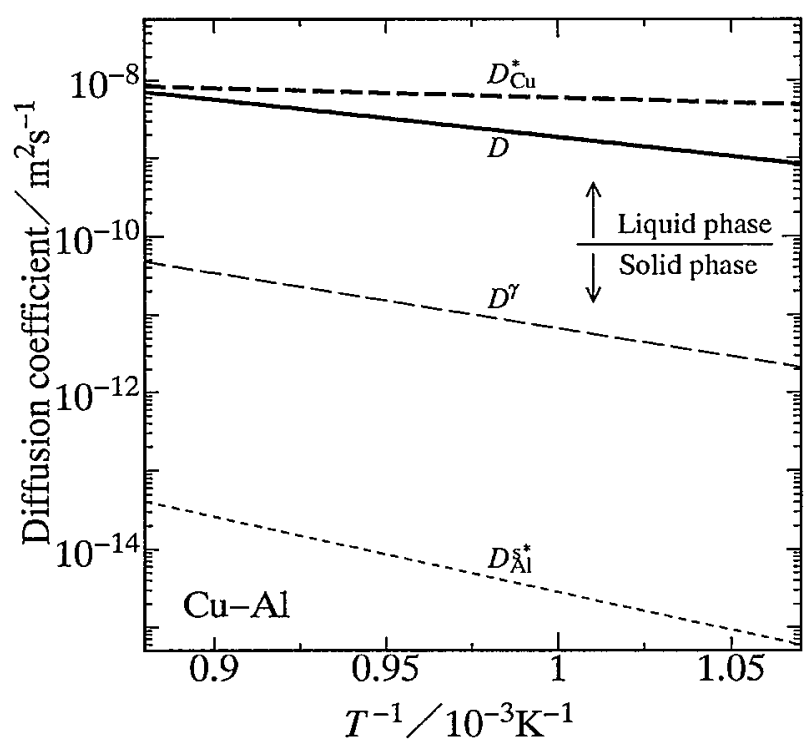

Fig. 7 Various diffusion coefficients versus the reciprocal of the temperature $T$ shown as different straight lines. experiment, the diffusion couple was isothermally annealed at temperatures between $T=673$ and $808 \mathrm{~K}$. During annealing, compound layers of the $\gamma, \delta, \zeta, \eta$ and $\theta$ phases are formed at each interface in the diffusion couple. According to the observation, the parabolic relationship holds good between the mean thickness of each compound layer and the annealing time. This means that the growth of the compound layer is controlled by volume diffusion. From the experimental values of the parabolic coefficient at various annealing temperatures, the temperature dependence of the interdiffusion coefficient was estimated for each compound. The estimation gives $D_{0}^{\gamma}=8.5 \times 10^{-5} \mathrm{~m}^{2} / \mathrm{s}$ and $Q^{\gamma}=136$ $\mathrm{kJ} / \mathrm{mol}$ as the pre-exponential factor and the activation enthalpy, respectively, of the interdiffusion coefficient $D^{\gamma}$ for the $\gamma$ phase. ${ }^{42)}$ The temperature dependence of $D^{\gamma}$ with these parameters is shown as a thin dashed line in Fig. 7. Since the $\beta$ and $\varepsilon$ phases are not stable at $T=673-808 \mathrm{~K},{ }^{37)}$ the interdiffusion coefficient was not determined for these compounds in their experiment. On the other hand, the preexponential factor $D_{\mathrm{Al} 0}^{\mathrm{s} *}$ and the activation enthalpy $Q_{\mathrm{Al}}^{\mathrm{s*}}$ of the tracer diffusion coefficient $D_{\mathrm{Al}}^{\mathrm{s} *}$ of $\mathrm{Al}$ in pure $\mathrm{Cu}$ were reported as follows: $D_{\mathrm{Al} 0}^{\mathrm{s} *}=1.31 \times 10^{-5} \mathrm{~m}^{2} / \mathrm{s}$ and $Q_{\mathrm{Al}}^{\mathrm{s} *}=$ $185 \mathrm{~kJ} / \mathrm{mol}^{43)}$ The temperature dependence of $D_{\mathrm{Al}}^{\mathrm{s} *}$ with these parameters is shown as a thin dotted line in Fig. 7. As can be seen, both $D_{\mathrm{Al}}^{\mathrm{s} *}$ and $D^{\gamma}$ are much smaller than $D$. Hence, we may expect that the interdiffusion coefficient is much smaller also for the $\beta$ and $\varepsilon$ phases than for the $L$ phase. Consequently, it is concluded that the diffusional flux is much smaller in the $\alpha, \beta, \gamma$ and $\varepsilon$ phases than in the $L$ phase during reactive diffusion in the $\mathrm{Cu} / \mathrm{Al}$ diffusion couple at $T=$ $973-1073 \mathrm{~K}$. This is the reason why the $L / \varepsilon$ interface migrates towards the $\varepsilon$ phase and the migration rate of the interface is much greater than the growth rates of the $\beta, \gamma$ and $\varepsilon$ layers.

\subsection{Penetration depth of interdiffusion}

At each annealing temperature, the mol fraction $y$ of $\mathrm{Al}$ in the $L$ phase is expressed as a function of the distance $x$ and the annealing time $t$ by the following equation. ${ }^{39)}$

$$
y=y^{L 0}+\frac{y^{L \varepsilon}-y^{L 0}}{1+\operatorname{erf}\left(K_{w}\right)}\left\{1+\operatorname{erf}\left(\frac{x}{\sqrt{4 D t}}\right)\right\}, \quad x<w
$$

Here, $x$ is measured from the initial position of the $\mathrm{Cu} / \mathrm{Al}$ interface in the diffusion couple. From eq. (19), $y$ was calculated as a function of $x$ for the longest annealing time of $t=2.4 \times 10^{3} \mathrm{~s}(40 \mathrm{~min})$ using the following parameters as well as $y^{L 0}=1$ and the value of $y^{L \varepsilon}$ obtained from eq. (16): $K_{w}=0.288$ and $D=1.24 \times 10^{-9} \mathrm{~m}^{2} / \mathrm{s}$ at $T=973 \mathrm{~K} ; K_{w}=$ 0.319 and $D=2.91 \times 10^{-9} \mathrm{~m}^{2} / \mathrm{s}$ at $T=1023 \mathrm{~K}$; and $K_{w}=$ 0.349 and $D=3.62 \times 10^{-9} \mathrm{~m}^{2} / \mathrm{s}$ at $T=1073 \mathrm{~K}$. The results of $T=973,1023$ and $1073 \mathrm{~K}$ are shown as thin dashed curves in Fig. 8(a), (b) and (c), respectively. In this figure, the ordinate and the abscissa indicate the mol fraction $y$ and the distance $x$, respectively. As long as $y$ is equal to $y^{L 0}$ at $x=x_{\mathrm{s}}$, the $\mathrm{Cu} / \mathrm{Al}$ diffusion couple is considered semi-infinite during annealing. Here, $x_{\mathrm{s}}$ is the position of the flat surface of the $L$ phase parallel to the interface. The initial thickness of the $L$ phase is $4.8 \mathrm{~mm}$ and the $L$ phase is initially located on the negative side of $x$ in Fig. 8, and thus $x_{\mathrm{s}}$ is equal to $-4.8 \mathrm{~mm}$. As can be seen in Fig. 8, however, the penetration depth of 


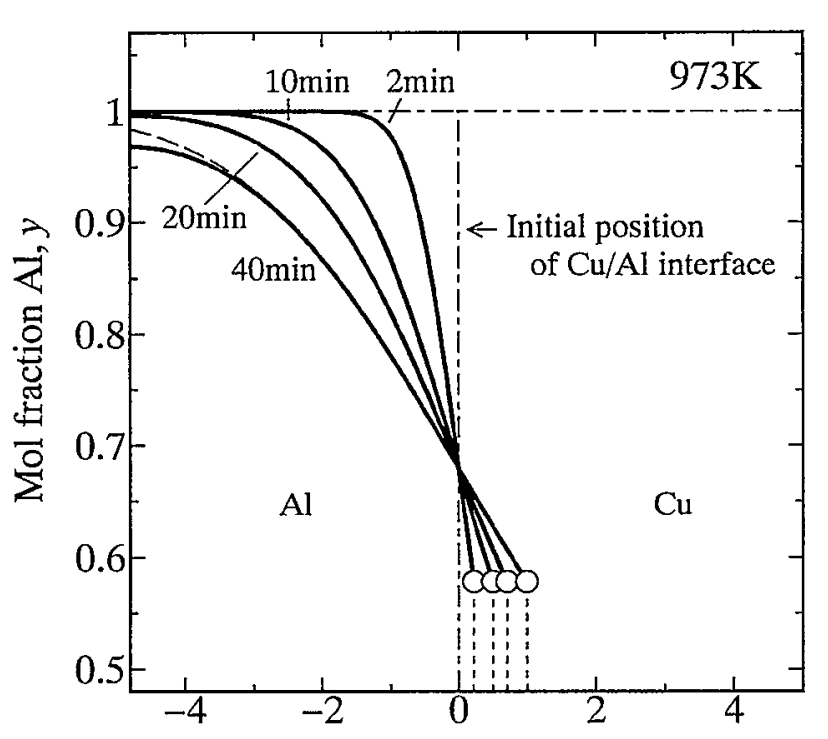

(a)

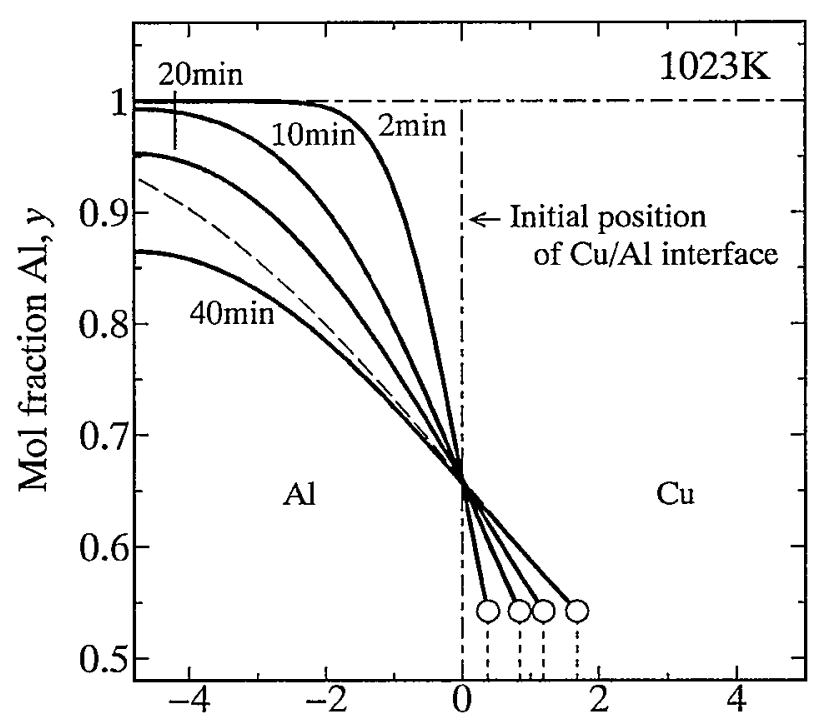

(b)

Distance, $x / 10^{-3} \mathrm{~m}$

interdiffusion in the $L$ phase exceeds the thickness of the $L$ phase at $t=2.4 \times 10^{3} \mathrm{~s}(40 \mathrm{~min})$ for all the annealing temperatures, and hence $y$ becomes smaller than $y^{L 0}$ at $x=x_{\mathrm{s}}$. Consequently, at the longest annealing time, the $\mathrm{Cu}$ / $\mathrm{Al}$ diffusion couple is no longer semi-infinite. In such a case, eq. (19) is not applicable. In order to calculate the concentration profile in the $L$ phase for various annealing times up to the longest time, eqs. (7) and (10) were solved numerically under the following initial and boundary conditions:

$$
\begin{aligned}
& y^{L}(x<0, t=0)=y^{L 0}, \\
& y^{L}(x=w, t>0)=y^{L \varepsilon}, \\
& y^{\alpha}(x=w, t>0)=y^{\alpha 0}
\end{aligned}
$$

and

$$
\left.\frac{\partial y^{L}}{\partial x}\right|_{x=x_{\mathrm{s}}}=0 .
$$

In the present numerical calculation, Crank-Nicolson implicit method $^{44)}$ was combined with a finite-difference technique. ${ }^{45)}$ The results of $T=973,1023$ and $1073 \mathrm{~K}$ are shown as bold

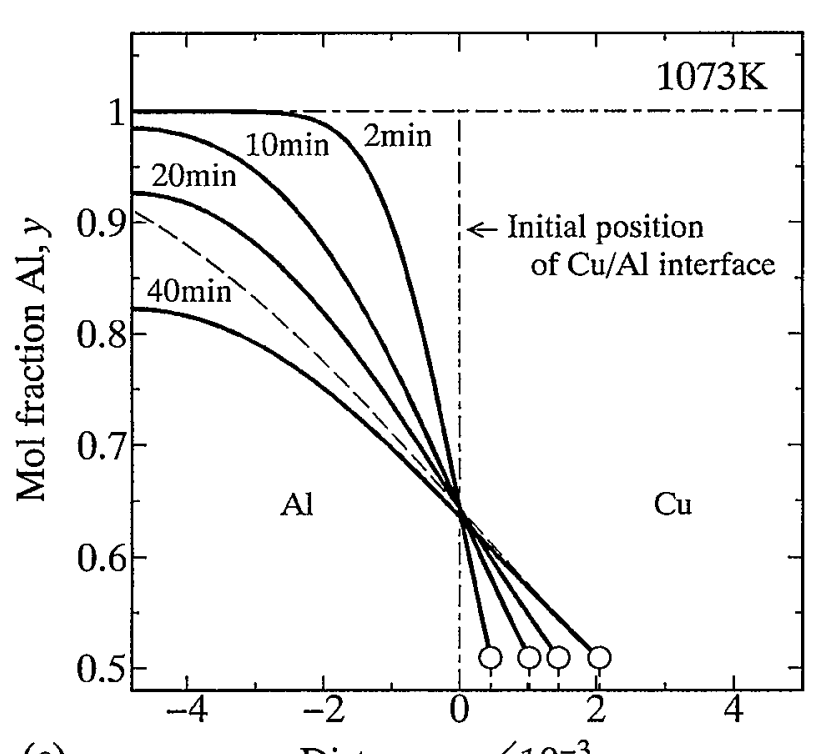

(c)

Distance, $x / 10^{-3} \mathrm{~m}$
Fig. 8 Concentration profiles of $\mathrm{Al}$ in the $L$ phase calculated from eq. (19) are shown as thin dashed curves for (a) $T=973 \mathrm{~K}$, (b) $T=1023 \mathrm{~K}$ and (c) $T=1073 \mathrm{~K}$. Solid curves indicate the numerical calculations from eqs. (7) and (10) under the initial and boundary conditions of eq. (20). solid curves in Fig. 8(a), (b) and (c), respectively. Open circles indicate the composition and the position of the interface at different annealing times. At $T=973 \mathrm{~K}$ in Fig. 8(a), the penetration depth is smaller than the thickness of the $L$ phase at $t=1.2 \times 10^{2}$ and $6.0 \times 10^{2} \mathrm{~s}(2$ and $10 \mathrm{~min})$ but greater than that of the $L$ phase at $t=1.2 \times 10^{3}$ and $2.4 \times 10^{3} \mathrm{~s}$ (20 and $\left.40 \mathrm{~min}\right)$. In contrast, at $T=1023$ and $1073 \mathrm{~K}$ in Fig. 8(b) and (c), the penetration depth outstrips the thickness of the $L$ phase even at $t=6.0 \times 10^{2} \mathrm{~s}(10 \mathrm{~min})$. The annealing time dependence of the mol fraction $y_{\mathrm{s}}$ at $x=x_{\mathrm{s}}$ was deduced from the numerical calculation in Fig. 8 . The results of $T=973,1023$ and $1073 \mathrm{~K}$ are shown as dotted, dashed and solid curves, respectively, in Fig. 9. In this figure, the ordinate indicates the mol fraction $y_{\mathrm{s}}$, and the abscissa shows the square root of the annealing time $t$. As can be seen, $y_{\mathrm{s}}$ is equal to $y^{L 0}$ at short annealing times. In this case, the $\mathrm{Cu} / \mathrm{Al}$ diffusion couple is considered semi-infinite. At long annealing times, however, $y_{\mathrm{s}}$ becomes smaller than $y^{L 0}$, and hence the diffusion couple is no longer semi-infinite. If the $\mathrm{Cu} / \mathrm{Al}$ diffusion couple is not semi-infinite, the 


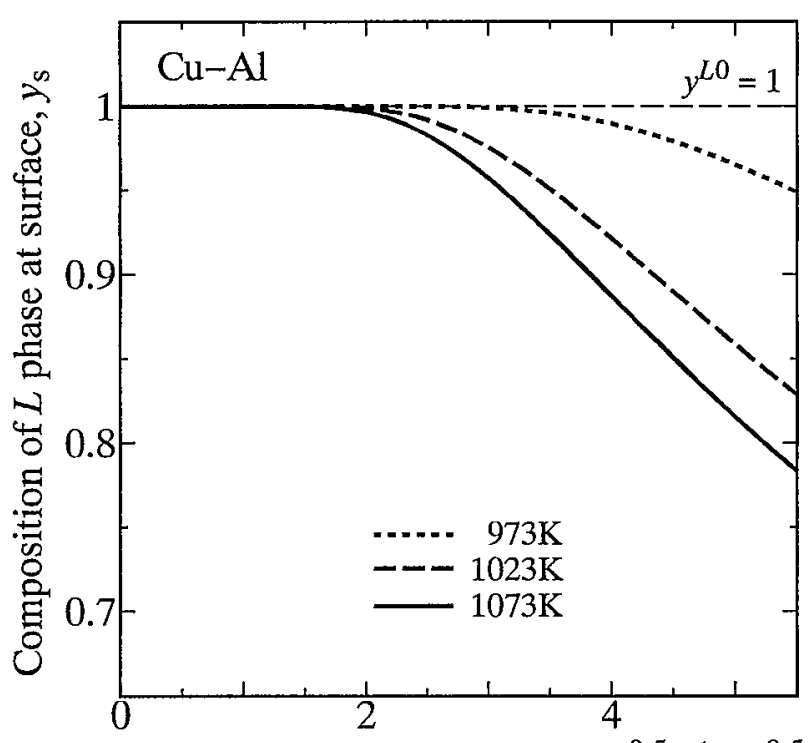

Square root of annealing time, $t^{0.5} / 10 \mathrm{~s}^{0.5}$

Fig. 9 The composition $y_{\mathrm{s}}$ at $x=x_{\mathrm{s}}$ versus the square root of the annealing time $t$ for the numerical calculation in Fig. 8 shown as dotted, dashed and solid curves at $T=973,1023$ and $1073 \mathrm{~K}$, respectively.

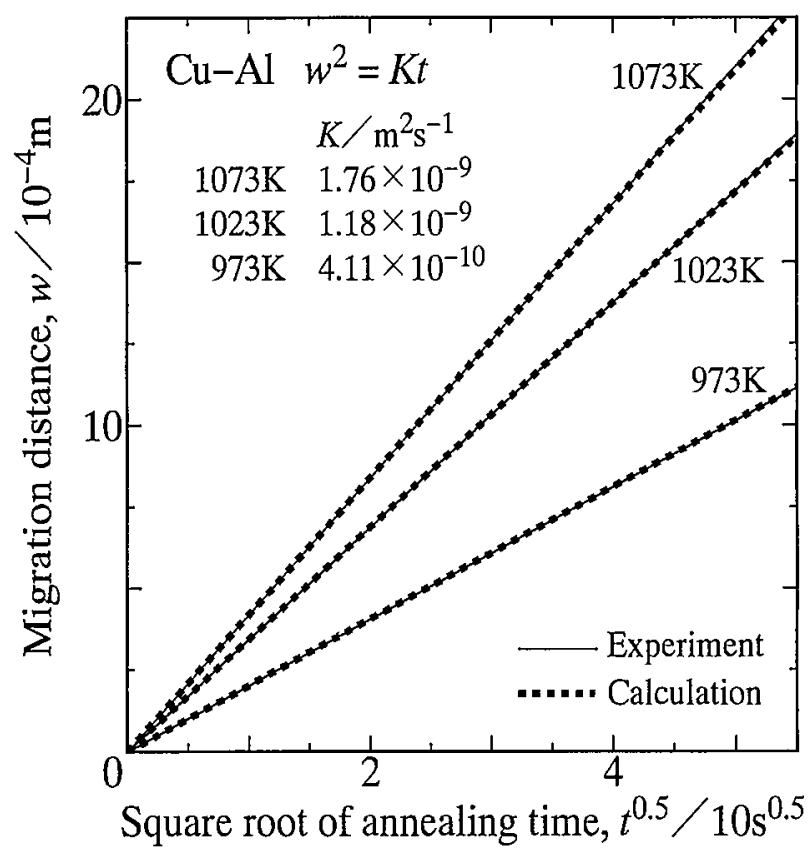

Fig. 10 Straight lines in Fig. 2 are represented as thin solid lines. The corresponding results deduced from the numerical calculation in Fig. 8 are shown as bold dotted curves.

parabolic relationship may not hold good even for the migration of the interface controlled by volume diffusion. Nevertheless, most of the plotted points at each annealing temperature are located well on the corresponding straight line in Fig. 2. The straight lines in Fig. 2 are indicated again as thin solid lines in Fig. 10. On the other hand, the relationship between the migration distance $w$ and the annealing time $t$ was derived from the numerical calculation in Fig. 8. The results of $T=973,1023$ and $1073 \mathrm{~K}$ are shown as bold dotted curves in Fig. 10. As can be seen, the dotted curve coincides well with the solid line at $T=973 \mathrm{~K}$. Also at $T=1023$ and $1073 \mathrm{~K}$, the dotted curves mostly consist with the corresponding solid lines. As shown in Fig. 8(a), at $T=973 \mathrm{~K}$, the dashed curve agrees with the solid curve of $t=2.4 \times 10^{3} \mathrm{~s}(40 \mathrm{~min})$ except at small values of $x$. Even at $T=1023$ and $1073 \mathrm{~K}$, the dashed curves accord with the solid curves of $t=2.4 \times 10^{3} \mathrm{~s}(40 \mathrm{~min})$ in the neighborhood of the interface as indicated in Fig. 8(b) and (c), respectively. In Fig. 8, the dashed and solid curves show the concentration profiles for the semi-infinite and finite diffusion couples, respectively. According to eq. (10), the migration rate of the interface is determined by the concentration gradient in the $L$ phase at the interface. As can be seen in Fig. 8, the concentration gradient at the interface is mostly equivalent for the semi-infinite and finite diffusion couples even at the longest annealing time. Therefore, almost the same migration rate of the interface is realized in both diffusion couples under the present annealing conditions. This is the reason why the parabolic relationship holds good within experimental uncertainty also at the long annealing times where the diffusion couple is no longer semi-infinite. When migration of an interface is controlled by volume diffusion in a semi-infinite diffusion couple, the migration surely obeys the parabolic relationship. However, the present numerical calculation indicates that the parabolic relationship holds good even for a finite diffusion couple as long as volume diffusion is the rate-controlling process and the penetration depth of interdiffusion merely slightly eclipses the thickness of each specimen in the diffusion couple.

\section{Conclusions}

The kinetics of the reactive diffusion in the binary $\mathrm{Cu}-\mathrm{Al}$ system was experimentally observed in a previous study. ${ }^{28)}$ In that experiment, $\mathrm{Cu} / \mathrm{Al}$ diffusion couples initially consisting of pure $\mathrm{Cu}$ and $\mathrm{Al}$ were isothermally annealed at temperatures of $T=973-1073 \mathrm{~K}$. At these temperatures, $\mathrm{Cu}$ is solid, but $\mathrm{Al}$ is liquid. During annealing, compound layers of the $\beta, \gamma$ and $\varepsilon$ phases $^{37)}$ are formed between the $\mathrm{Cu}$-rich solid $(\alpha)$ phase and the Al-rich liquid $(L)$ phase in the diffusion couple. Furthermore, the $L / \varepsilon$ interface migrates towards the $\varepsilon$ phase. Between the migration distance $w$ of the $L / \varepsilon$ interface and the annealing time $t$, there exists the parabolic relationship $w^{2}=K t$, where $K$ is the parabolic coefficient. The observation provides $K=4.11 \times 10^{-10}$, $1.18 \times 10^{-9}$ and $1.76 \times 10^{-9} \mathrm{~m}^{2} / \mathrm{s}$ at $T=973,1023$ and $1073 \mathrm{~K}$, respectively. The total thickness $l$ of the compound layers is much smaller than the migration distance $w$. This means that the migration of the $L / \varepsilon$ interface is predominantly controlled by the volume diffusion in the $L$ phase. In order to evaluate the interdiffusion coefficient $D$ of the $L$ phase, the experimental results were mathematically analyzed using the diffusion equation describing the flux balance at the migrating interface. ${ }^{39}$ ) The analysis deduces $D=$ $1.24 \times 10^{-9}, 2.91 \times 10^{-9}$ and $3.62 \times 10^{-9} \mathrm{~m}^{2} / \mathrm{s}$ at $T=973$, 1023 and $1073 \mathrm{~K}$, respectively. When the temperature dependence of $D$ is expressed by the equation $D=$ $D_{0} \exp (-Q / R T)$, values of $D_{0}=1.42 \times 10^{-4} \mathrm{~m}^{2} / \mathrm{s}$ and $Q=93.5 \mathrm{~kJ} / \mathrm{mol}$ are obtained by the least-squares method. According to the analysis, the interdiffusion occurs much faster in the $L$ phase than in the solid phases. This is the 
reason why the $L / \varepsilon$ interface migrates towards the $\varepsilon$ phase and the migration rate of the interface is much greater than the overall growth rate of the compound layers.

\section{Acknowledgements}

The present study was supported by a Grant-in-Aid for Scientific Research from the Ministry of Education, Culture, Sports, Science and Technology of Japan.

\section{REFERENCES}

1) T. B. Massalski, H. Okamoto, P. R. Subramanian and L. Kacprzak: Binary Alloy Phase Diagrams (ASM International, Materials Park, $\mathrm{OH}$, 1990) vol. 1-3.

2) B. Lustman and R. F. Mehl: Trans. Met. Soc. AIME 147 (1942) 369394.

3) D. Horstmann: Stahl Eisen 73 (1953) 659-665.

4) S. Storchheim, J. L. Zambrow and H. H. Hausner: Trans. Met. Soc. AIME 200 (1954) 269-274.

5) G. V. Kidson and G. D. Miller: J. Nucl. Mater. 12 (1964) 61-69.

6) K. Shibata, S. Morozumi and S. Koda: J. Japan Inst. Met. 30 (1966) 382-388.

7) K. Hirano and Y. Ipposhi: J. Japan Inst. Met. 32 (1968) 815-821.

8) M. M. P. Janssen: Metall. Trans. 4 (1973) 1623-1633.

9) G. F. Bastin and G. D. Rieck: Metall. Trans. 5 (1974) 1817-1826.

10) M. Onishi and H. Fujibuchi: Trans. JIM 16 (1975) 539-547.

11) EI-B. Hannech and C. R. Hall: Mater. Sci. Tech. 8 (1992) 817-824.

12) P. T. Vianco, P. F. Hlava and A. L. Kilgo: J. Electron. Mater. 23 (1994) 583-594.

13) M. Watanabe, Z. Horita and M. Nemoto: Interface Science 4 (1997) 229-241.

14) S. Choi, T. R. Bieler, J. P. Lucas and K. N. Subramanian: J. Electron. Mater. 28 (1999) 1209-1215.

15) M. Kajihara, T. Yamada, K. Miura, N. Kurokawa and K. Sakamoto: Netsushori 43 (2003) 297-298.

16) T. Yamada, K. Miura, M. Kajihara, N. Kurokawa and K. Sakamoto: J. Mater. Sci. 39 (2004) 2327-2334.

17) T. Yamada, K. Miura, M. Kajihara, N. Kurokawa and K. Sakamoto: Mater. Sci. Eng. A 390 (2005) 118-126.

18) K. Suzuki, S. Kano, M. Kajihara, N. Kurokawa and K. Sakamoto:
Mater. Trans. 46 (2005) 969-973.

19) M. Mita, M. Kajihara, N. Kurokawa and K. Sakamoto: Mater. Sci. Eng. A 403 (2005) 269-275.

20) T. Takenaka, S. Kano, M. Kajihara, N. Kurokawa and K. Sakamoto: Mater. Sci. Eng. A 396 (2005) 115-123.

21) D. Naoi: Master Eng. Thesis, Tokyo Institute of Technology, 2006.

22) T. Takenaka, M. Kajihara, N. Kurokawa and K. Sakamoto: Mater. Sci. Eng. A 406 (2005) 134-141.

23) T. Takenaka, S. Kano, M. Kajihara, N. Kurokawa and K. Sakamoto: Mater. Trans. 46 (2005) 1825-1832.

24) M. Mita, K. Miura, T. Takenaka, M. Kajihara, N. Kurokawa and K. Sakamoto: Mater. Sci. Eng. B 126 (2006) 37-43.

25) T. Takenaka and M. Kajihara: Mater. Trans. 47 (2006) 822-828.

26) T. Takenaka, M. Kajihara, N. Kurokawa and K. Sakamoto: Mater. Sci. Eng. A 427 (2006) 210-222.

27) Y. Yato and M. Kajihara: Mater. Sci. Eng. A 428 (2006) 276-283.

28) Y. Tanaka, M. Kajihara and Y. Watanabe: Mater. Sci. Eng. A, in press.

29) Y. Yato and M. Kajihara: Mater. Trans. 47 (2006) 2277-2284.

30) Y. Muranishi and M. Kajihara: Mater. Sci. Eng. A 404 (2005) 33-41.

31) T. Hayase and M. Kajihara: Mater. Sci. Eng. A 433 (2006) 83-89.

32) M. Kajihara: Acta Mater. 52 (2004) 1193-1200.

33) M. Kajihara: Mater. Sci. Eng. A 403 (2005) 234-240.

34) M. Kajihara: Mater. Trans. 46 (2005) 2142-2149.

35) M. Kajihara: Defect and Diffusion Forum 249 (2006) 91-95.

36) M. Kajihara: Mater. Trans. 47 (2006) 1480-1484.

37) T. B. Massalski, H. Okamoto, P. R. Subramanian and L. Kacprzak: Binary Alloy Phase Diagrams (ASM International, Materials Park, OH, 1990) vol. 1, pp. 141-143.

38) Y. L. Corcoran, A. H. King, N. de Lanerolle and B. Kim: J. Electron. Mater. 19 (1990) 1177-1183.

39) W. Jost: Diffusion of Solids, Liquids, Gases (Academic Press, New York, 1960) pp. 68-82.

40) G. Dahlquist and A. Björck: Numerical Methods (Prentice-Hall, Englewood Cliffs, NJ, 1974) pp. 222-224.

41) T. Ejima, T. Yamamura, N. Uchida, Y. Matsuzaki and M. Nikaido: J. Jpn. Inst. Met. 44 (1980) 316-323.

42) Y. Funamizu and K. Watanabe: Trans. Jpn. Inst. Met. 12 (1971) 147152.

43) Metals Data Book, ed. Japan Institute of Metals (Maruzen, Tokyo, 1993) p. 21.

44) J. Crank: The Mathematics of Diffusion (Oxford Univ. Press, London, 1979) pp. 144-148.

45) R. A. Tanzilli and R. W. Heckel: Trans. Met. Soc. AIME 242 (1968) 2313-2321. 